\title{
Correction to: Paraburkholderia atlantica sp. nov. and Paraburkholderia franconis sp. nov., two new nitrogen-fixing nodulating species isolated from Atlantic forest soils in Brazil
}

\author{
Fabiane Paulitsch ${ }^{1,2,3} \cdot$ Rebeca Fuzinatto Dall'Agnol ${ }^{1,3}$. Jakeline Renata Marçon Delamuta ${ }^{1,4}$. \\ Renan Augusto Ribeiro ${ }^{4}$ Jesiane Stefania da Silva Batista ${ }^{5}$. Mariangela Hungria ${ }^{1,2,4}$
}

Published online: 21 May 2021

(c) Springer-Verlag GmbH Germany, part of Springer Nature 2021

\section{Correction to: \\ Archives of Microbiology (2020) 202:1369-1380 \\ https://doi.org/10.1007/s00203-020-01843-w}

The original article has published with few errors. The corrections are as follows.

The type strain is CNPSo $3155^{\mathrm{T}}$ (= ABIP $236^{\mathrm{T}},=\mathrm{LMG} 31643^{\mathrm{T}}$ ), isolated from nodules of Mimosa pudica grown in soil of the Brazilian Atlantic Forest, Rio de Janeiro State, Brazil.

In the supplementary material.

CNPSo $3155^{\mathrm{T}}$ corresponding to ABIP $236^{\mathrm{T}}$, LMG31643 ${ }^{\mathrm{T}}$.
Paraburkholderia atlantica (at.lan'ti.ca. L. fem. adj. atlantica pertaining to the Atlantic Forest biome of Brazil "Mata Atlântica").

Publisher's Note Springer Nature remains neutral with regard to jurisdictional claims in published maps and institutional affiliations.
The original article can be found online at https://doi.org/10.1007/ s00203-020-01843-w.

Mariangela Hungria

mariangela.hungria@embrapa.br

Fabiane Paulitsch

fabi_paulitsch@hotmail.com

Rebeca Fuzinatto Dall'Agnol rebeca.fd@hotmail.com

Jakeline Renata Marçon Delamuta jake_renata@hotmail.com

Renan Augusto Ribeiro renanribeiro83@hotmail.com

Jesiane Stefania da Silva Batista jesiane.batista@gmail.com
Embrapa Soja, C.P. 231, Londrina, Paraná 86001-970, Brazil

2 Departamento de Microbiologia, Universidade Estadual de Londrina, C.P. 10011, Londrina, Paraná 86057-970, Brazil

3 Coordenação de Aperfeiçoamento de Pessoal de Nível Superior, SBN, Quadra 2, Bloco L, Lote 06, Edifício Capes, Brasília, Distrito Federal 70.040-020, Brazil

4 Conselho Nacional de Desenvolvimento Científico E Tecnológico, SHIS QI 1 Conjunto B, Blocos A, B, C e D, Lago Sul, Brasília, Distrito Federal 71605-001, Brazil

5 Departamento de Biologia Estrutural, Molecular E Genética, Universidade Estadual de Ponta Grossa, Avenida General Carlos Cavalcanti, 4748 - Uvaranas, C.P. 6001, Ponta Grossa, PR 84030-900, Brazil 